\author{
Review Article \\ www.ijrap.net (ISSN:2229-3566)
}

\section{A REVIEW ON SKIN MICROBIOME: NOVEL STRATEGY IN COSMETICS} \\ Disha P Prajapati ${ }^{1 *}$, Tanvi R Dodiya ${ }^{2}$ \\ ${ }^{1}$ Assistant Professor, Parul Institute of Pharmacy and Research, Parul University, Vadodara, Gujarat, India \\ ${ }^{2}$ Associate Professor, Parul Institute of Pharmacy and Research, Parul University, Vadodara, Gujarat, India
}

Received on: 25/05/21 Accepted on: 25/06/21

\begin{abstract}
*Corresponding author
E-mail: disha.prajapati@paruluniversity.ac.in
\end{abstract}

DOI: 10.7897/2277-4343.120382

\begin{abstract}
Human skin is the largest organ composing a complex ecosystem harbouring different types of microorganisms such as bacteria, viruses, fungi and mites that are together known as the skin microbiome. These organisms play an important role in communicating and instructing the cutaneous arm of the immune system to keep the skin healthy. Diet, hormonal imbalance, lifestyle, use of medications and cosmetics have been reported to influence the composition of skin microbiome. These factors can sometimes cause an imbalance in the microbiome leading to a condition known as 'dysbiosis'. This disruption can then lead to several skin disorders such as dandruff, acne, psoriasis, or atopic dermatitis. Hygiene products, make-up, perfume, skin cream, nail polish, soap, shampoo, shaving cream, deodorant etc. also implicated in modifying the skin microbiome. Consumers today are moving towards a simpler, cleaner and nature based products for cosmetics. Microbiome balancing skincare natural products can respond to the rising concerns of environment pollution, skin sensitivity and premature ageing. These products can enrich the skin to repair itself by boosting skins immune system and natural defence barrier. These natural products are meant to remove pathogenic but to retain the composition mutualistic organism. The skincare market has recently embraced these distinct approaches of targeting the skin microbiome through different fronts like Probiotics, Prebiotics and Postbiotics.
\end{abstract}

Keywords: Skin microbiome, prebiotics, probiotics, and cosmetics.

\section{INTRODUCTION}

Skin is considered to be the largest organ of the human body with an average surface area of 1.6-2 sq. meters and accounts for about $15 \%$ of the total body weight of an adult human ${ }^{1}$. It is an interface with the environment and protects the body against pathogens, controls water loss, regulates the body temperature, enables sensation to be perceived ${ }^{2}$.

The skin is divided into three layers namely, epidermis which is a stratified, squamous epithelium layer that is composed primarily of two types of cells: keratinocytes and dendritic cells. The keratinocytes differ from the clear dendritic cells by possessing intercellular bridges and ample amounts of stainable cytoplasm. The epidermis harbours a number of other cell populations, such as Melanocytes, Langerhans cells and Merkel cells, but the Keratinocyte cell type comprises the majority of the cells by far. Second is the basement membrane which is the multi-layered structure forming the dermo epidermal junction. The interface between the epidermis and dermis is formed by a porous basement membrane zone that allows the exchange of cells and fluid and holds the two layers together and lastly the dermis which is an integrated system of fibrous, filamentous and amorphous connective tissue that accommodates stimulus-induced entry by nerve and vascular networks, epidermally derived appendages, fibroblasts, macrophages and mast cells ${ }^{3}$. The dermis is followed by hypodermis which is the layer of loose connective tissue and fat beneath the dermis ${ }^{4}$.

\section{Skin microbiome}

Recently, a lot of buzz has been created by the word "microbiome" coined by Nobel Laureate Joshua Lederberg.
Microbiome is defined as the entire collection of microorganisms living either inside or outside of your body. After completion of the Human Microbiome Project in 2012, carried out by the US National Institutes of Health, the knowledge about the types and the importance of these microbes for humans came to light. Since then, it has been a hot topic in healthcare, skincare, and even the food and beverage industry, with current estimates placing the global market value of the microbiome industry at $\$ 1.5$ billion USD by $2025^{5}$.

The role of the microbiome in the gut is well established and has been a vital topic in the field of healthcare and nutrition. However, information on the microbes that are lurking on the surface of the skin is new and is being labelled as the latest trend in medical science and the beauty industry. According to reports by Mintel, there have been huge advances in utilization of the skin microbiome in the cosmetics market, with growth estimated at a global CAGR of $6 \%$ between 2016 and 2021. It was also reported that the use of Lactobacillus-based probiotic skincare products increased by $98 \%$ in the United States from 2013 to 2017. Moreover, some of the key players in the cosmetics industry such as Procter \& Gamble, Johnson \& Johnson, BASF and Loreal have already turned their attention toward harnessing the full potential of the skin microbiome ${ }^{6}$.

Human skin is the largest organ composing a complex ecosystem harbouring different types of microorganisms such as bacteria, viruses, fungi and mites that are together known as the skin microbiome. More specifically these microbes colonize the Stratum corneum of the epidermis and skin appendages such as sweat glands and hair follicles ${ }^{7}$. 


\section{The skin microbiome includes two groups}

\section{Resident microorganisms (the core microbiome)}

Normal resident flora is microorganisms that are always present on or in a person and usually do not cause any disease. These are the permanent residents in the skin depending on the part of the body. The normal flora on skin is staphylococci and in mouth there are streptococci. Healthy people live in harmony with most of the microorganisms that establish themselves on or in (colonize) nonsterile parts of the body, such as the skin, nose, mouth, throat, large intestine, and vagina. The microorganisms that usually occupy a particular body site are called the resident flora.

\section{Transient microorganisms (the 'tourists')}

This microbiome colonizes the host for a short period of time, they sometimes are harmless or sometimes may even cause skin disease like yeast candida. Microorganisms that colonize people for hours to weeks but do not establish themselves permanently are called transient flora ${ }^{8}$.

\section{Alterations of skin microbiome according to age}

The skin microbiome is present from birth to death of the individual. The skin of infant is colonized by microorganisms when they are born and continues to explore surrounding environment ${ }^{9}$. During the infancy it is important to educate the immune system and develop susceptibility to allergic diseases by exposure to infectious agents, symbiotic microorganisms and parasites, etc ${ }^{10}$.

When they enter puberty there is production of more sebum by sebaceous gland due to increase in hormones. This condition can lead to colonization and growth of lipophilic microorganism Propionibacterium acnes. The change in this microbial composition can cause a common skin disease- acne vulgaris affecting $85 \%$ of adolescents and $11 \%$ of the adults ${ }^{11}$.

When teenagers enter the adulthood their skin microbiome reaches the equilibrium. But during the adulthood, the level of stress is mounting in life of an individual which has remarkable impact on the skin condition. Skin being the largest neuroendocrine organ; it has its own HPA (Hypothalamic pituitary adrenal) axis to produce cortisol under stress condition. A healthy skin barrier is affected by cortisol which alters the growth of commensal S. epidermises which inhibit the growth of pathogenic $S$. aureus. This alteration in the skin microorganism can develop Atopic dermatitis which is a chronic inflammatory skin disease characterized by defective skin barrier and colonization of $S$. aureus ${ }^{12-15}$.

As the person grows old the skin microbiome gets altered again. There is more colonization of Corynebacterium and decrease in Propionibacterium in old individuals. Thus, an aged skin can provide more advantageous ecosystem to certain microbes than others $^{16}$.

\section{The common microorganisms found in the skin are}

\section{Propionibacterium acnes}

They are found on the oily surface of the skin and hair follicles. They contribute to the development of acne as they proliferate due to excess oil production and clogged pores. They use sebum as fuel.

\section{Corynebacterium}

They are of two types pathogenic and non-pathogenic bacteria causing diphtheria which is inflammation in the throat and mucous membrane of nose and some nondiptherial infections can cause meningitis and urinary tract infection.

\section{Staphylococcus epidermidis}

They are harmless inhabitants of skin. They form a thick biofilm barrier which is a slimy substance that protects bacteria from antibiotics, chemicals and other substance or conditions that are hazardous.

\section{Staphylococcus aureus}

They are found on the skin and mucous membranes of humans. They are usually harmless but infections can occur on broken skin or within a blocked sweat or sebaceous gland.

\section{Streptococcus pyogenes}

These bacteria cause skin infection like impetigo, abscess, broncho-pulmonary infections and can cause acute articular rheumatism ${ }^{16-18}$.

\section{Factors contributing to variation in the skin microbiome}

The ecology of the skin surface is influenced by many variables including lifestyle, age, emotion, community, genetics, diet, hygiene products and drugs ${ }^{19-20}$.

Skin disorders such as atopic dermatitis, dandruff and vitiligo also affect the subjects' skin microbiome. Age-related changes in skin structure and function are attributable to combinations of endogenous intrinsic factors, e.g., cellular metabolisms, immune activity, hormone condition and exogenous environmental factors, e.g., exposure to sun, pollutants, other toxins. Under favourable conditions, an altered community of microbes can arise on the skin thus leading to conditions of dysbiosis ${ }^{21}$. Dysfunction of the capacity to re-establish a normal microbial community may perpetuate chronic disease including physiological injury and psychological anxiety, promoting endocrine and metabolic changes within the cutaneous microenvironments that directly impact the metabolic requirements and pathogenicity of various microorganisms

\section{Why take advantage of skin microbiome}

The skin barrier and the microbiota act like a shield that protects the body against external aggressions. There is a balanced interplay between the host and resident and/or transient bacterial populations. This balance is continuously affected by intrinsic (host) and extrinsic (environmental) factors that alter the composition of skin microorganism communities and the host skin barrier function. Altering this equilibrium is called dysbiosis $^{22}$. A robust skin microbiome protects against infection or dysbiosis in much the same way a good gut microbiome does, by colonization resistance either by competing for nutrients, secreting chemicals against them or stimulating the skin's immune system and by maintaining relatively acidic environment, which inhibits growth of pathogens. The skin microbiome could lessen the effects of UV exposure by providing immune protection in the skin's cellular microenvironment ${ }^{23}$.

A balanced microbiome may help ward off acne, as the skin microbiome's profile could be as accurate as $85 \%$ in predicting the clinical status of the skin ${ }^{24-25}$. 
The major disadvantage of skin microbiome is found that even resident microbes can cause skin diseases and enter the blood system creating life-threatening diseases particularly in immune suppressed people.

\section{Disorders caused by disruption of skin microbiome}

The majority of microbiota which inhabit the skin is harmless or beneficial and crucial for host defence. The relationship is "mutualistically symbiotic". Some microbes are inherently harmful, pathogenicity only occurs when the balance of the ecosystem is disturbed, and diversity is decreased. This "perturbation" of the skin microbiome, or "dysbiosis", is responsible for many skin conditions including acne, eczema, dermatitis, rosacea, inflammation, psoriasis, general allergies, sunburn, athlete's foot and ringworm, wound healing, diabetic skin, leishmaniasis, conjunctivitis and skin cancer ${ }^{26}$.

\section{Requirement of consumer for skin care}

Consumers today are moving towards simpler, cleaner and nature-based products for cosmetics. Microbiome balancing skincare natural products can respond to the rising concerns of environment pollution, skin sensitivity and premature ageing ${ }^{27}$.

Cosmetic formulators have begun to explore the relationship between the human microbiome and healthy skin. These products can enrich the skin to repair itself by boosting skins immune system and natural defence barrier. These natural products are meant to remove pathogenic but retain the composition of commensal and mutualistic organisms. The skincare market has recently embraced this distinct approach of targeting the skin microbiome through different fronts like

\section{Probiotics}

Probiotics are good bacteria in the form of either live cultures or lysates to increase the number of favourable bacteria on the skin. They improve the gut barrier function and restore the hut microecology which helps to stimulate the immune system of host and restore the alterations in the inflammatory. A regular consumption of fermented dairy products is likely to improve the natural skin barrier function and improved its cosmetic appearance. In the case of diseases with some imbalance in microorganisms, such as impure skin/mild acne or dry skin/mild atopic dermatitis, probiotic represent an effective alternative to antibacterial products ${ }^{28}$. Cosmetic Industries dealing with personal care products have assessed probiotics as "bioactive ingredients" to help enhance the beauty as well as the function of the skin. These products range from topical applications like body lotion, anti-aging serum, soap, aftershave, wipes etc to ingestible products like probiotic drinks. Lactobacillus is the most common genus of bacteria used as the ingredient for preparation of various probiotic enzymes, ferment lysate, probiotic proteins, etc. Some bacterial metabolites like lactic acid, hyaluronic acid is also found in the list of ingredients of the probiotics. The proposed mechanism found from scientific papers suggest that the beneficial effects of probiotics in topical application may be improving barrier function of the epithelium layer or inhibiting the growth of pathogens by limiting the nutrients and producing antibiotics ${ }^{29}$.

\section{Prebiotics}

They are the food source for the good bacteria promoting their growth and rebalance the skin microflora. They are usually short chain oligosaccharides or polysaccharides ${ }^{30}$.
A combination of prebiotics and probiotics in skin care can be wonderful for improving skin's surface protection, ensuring skin to keep its barrier intact, smooth and healthy.

\section{Postbiotics}

Postbiotics are waste (such as dead bacterial skin cells) from certain microorganisms, which by itself helps to improve the ecosystem of the skin. Like actual live probiotics, postbiotics also help nourish your skin. They are used along with pre and probiotics. They are beneficial in that they help support the growth of "good bacteria" and can even be anti-inflammatory, so they could potentially be of help with skin conditions such as acne and rosacea.

\section{Challenges in development of cosmetic containing Pro- prebiotic}

As the beauty products have their natural shelf life, the formulation of probiotic/prebiotic skin care products is different than the fresh foods used in ailments of digestive tract. It is difficult to add actual live bacteria in cosmetic, so most of them in fact contain fragments of bacterial cell walls that can reduce immune response.

One more challenge in using probiotic/prebiotic in the development of cosmetic is that there is diverse need of skin microflora of the individual and even the microflora present on different body parts is inherently different ${ }^{31-32}$.

\section{CONCLUSION}

The skin microbiome as a beauty concept is upending popular notions about bacteria's involvement in the skin. Beauty influencers are receptive to the idea of the skin as a living ecosystem which needs more natural, holistic and healthy beauty ${ }^{33}$. Currently, the cosmetic industry is mostly focused on prebiotic and probiotic solutions. Probiotics are commonly associated with gut health and live dairy products, yet it should be noted that when it comes to cosmetics, products contain extracts manufactured from bacteria rather than live cultures ${ }^{34}$. Some firms take a scientific approach with products that address specific concerns and benefits, such as wrinkle reduction, while others focus on nature, total skin health, holistic living and green beauty ${ }^{35}$. There is plenty of opportunity to innovate and also from skin microbiome-friendly cleansers and moisturizers to skin microbiome-enhancing probiotic mists and serums.

\section{REFERENCES}

1. Takanori I, Nishino K and Nayar SK. The appearance of human skin. Technical report: CUCS-024-05. Department of Computer Science Columbia University New York, USA; 2005.

2. Russell CW and Russell SW. Meta-analysis of skin microbiome: new link between skin microbiota diversity and skin health with proposal to use this as a future mechanism to determine whether cosmetic products damage the skin. Cosmetics 2017; 14: 1-19.

3. Paul AJ, Kolarsick BS and Maria A. Anatomy and physiology of skin. Journal of the Dermatology Nurses' Association 2011; 3: 203-213.

4. Heidi HK and Julia AS. Skin microbiome: looking back to move forward. Journal of Investigative Dermatology 2012; 132: 933-939.

5. Bincy B. Innovations in skin microbiome products: Saying yes to good bacteria. May 2019. Available from: 
https://www.prescouter.com/2019/05/innovations-skinmicrobiome-cosmetics/

6. Schommer NN and Gallo RL. Structure and function of the human skin microbiome. Trends in Microbiology 2013; 21: 680-688.

7. Elizabeth AG and Julia AS. The skin microbiome. National Review of Microbiology 2011; 9: 244-253.

8. Introduction to the Biology of Infectious Diseases - Infectious Diseases - MSD Manual Professional Edition https://www.msdmanuals.com/en-in/professional/infectiousdiseases/biology-of-infectious-disease/introduction-to-thebiology-of-infectious-diseases

9. Byrd AL, Belkaid Y and Segre AJ, The human skin microbiome. National Review of Microbiology; 2016. p. 143155.

10. Chrissy C. Pre/Probiotic skin care: what are they and do they work? Today; 2019. https://www.today.com/style/preprobiotic-skin-care-what-are-they-do-they-work-t149565

11. Nakako S, Wataru S, Cecile C, Philippe B, Lena T and Erica $\mathrm{I}$, et al. Aging-related changes in the diversity of women's skin microbiomes associated with oral bacteria. Scientific Reports 2017; 7 : 165-67.

12. Adam SM and Elizabeth AG. Interactions between host factors and the skin microbiome. Cellular and Molecular Life Sciences 2015; 72: 1499-1515.

13. Yan L. The human skin microbiome: a new way to beauty. Shanghai Pechoin Daily Chemical Co. Ltd. https://ifscc.org/wp-content/uploads/2019/08/2019-MaisonG-de-Navarre-winning-essay-Yan-Liu.pdf

14. Gibbon SF, Tomida S, Chiu BH, Nguyen L, Liu DC and Elashoff D, et al. Propionibacterium acnes strain populations in the human skin microbiome associated with acne. Journal of Investigational Dermatology 2013; 133: 2152-2160.

15. Sanford JA and Gallo RL. Functions of the skin microbiota in health and disease. Seminars in Immunology 2013; 25: 370 377.

16. Regina B. 5 Types of Bacteria That Live on Your Skin https://www.thoughtco.com/bacteria-that-live-on-your-skin373528; August 212019.

17. Jef Akst. Microbes of the Skin. The Scientist Magazine. https://www.the-scientist.com/news-analysis/microbes-ofthe-skin-37335; June 132014.

18. Barry L, Riley M, Kachiu CL, David JE and Lawrence E. The human skin microbiome. International Journal of Dermatology 2014; 53: 1177-1179.

19. Adam SM and Elizabeth AG. Interactions between host factors and the skin microbiome. Cellular and Molecular Life Sciences 2015; 72: 1499-1515.

20. Pedro AD, Brandon I, Kausar M, Hilary L, Mohn WW and Greg GH. New insights into the intrinsic and extrinsic factors that shape the human skin microbiome. Biology 2019; 839: $1-19$.

21. Scharschmidt TC, Fischbach MA. What Lives On Our Skin: Ecology, Genomics and Therapeutic Opportunities of the Skin Microbiome. Drug Discov Today Dis Mech 2013 Dec 1; 10(3-4): e83-e89. DOI: 10.1016/j.ddmec.2012.12.003. PMID: 24273587; PMCID: PMC3833721.
22. Dréno B, Araviiskaia E, Berardesca E, Gontijo G, Sanchez M and Viera LF et al. Microbiome in healthy skin, update for dermatologists. Journal of the European Academy of Dermatology and Venereology 2016; 30: 2038-2047.

23. Schleehauf B. It's time to take advantage of the skin microbiome.

https://www.cosmeticsandtoiletries.com/networking/eventco verage/Its-Time-to-Take-Advantage-of-the-Skin-

Microbiome-513260261.html; 2019.

24. Vijay KP, Karin W, Velmurugesan A and Peter W. Skin microbiome modulates the effect of ultraviolet radiation on cellular response and immune function. I science 2019; 15: 211-222.

25. Marc SR. Cosmetics: The next microbiome frontier. Chemical and Engineering news 2017; 95: 30-34.

26. Christopher WR. The role of every-day cosmetics in altering the skin microbiome: a study using biodiversity. Cosmetics 2019; 6: 2-22.

27. Beri K. Perspective: stabilizing the microbiome skin-gutbrain axis with natural plant botanical ingredients in cosmetics. Cosmetics 2018; 5: 2-8.

28. Marini A and Krutmann J. Pre- and Probiotics for human skin. Wageningen Academic Publishers; 2012. p. 318-331.

29. Huang MCJ and Tang J. Probiotics in personal care products. Microbiology Discovery 2015; 3: 1-9.

30. Simmering R and Breves RH. Pre and postbiotic. Cosmetics 2009; 60: 809-814.

31. Misery L, Loser K and Stander S. Sensitive skin. Journal of the European Academy of Dermatology and Venereology 2018; 30: 2-8.

32. Juge R, Rouaud TP and Breugnot J. Shift in skin microbiota of Western European women across aging. Journal of Applied Microbiology 2018; 125: 907-916.

33. Prescott SL, Larcombe DL, Logan AC, West C, Burks W, Caraballo L, Levin M, Etten EV, Horwitz P, Kozyrskyj A, Campbell DE. The skin microbiome: impact of modern environments on skin ecology, barrier integrity, and systemic immune programming. World Allergy Organ J. 2017 Aug 22; 10(1): 29. DOI: 10.1186/s40413-017-0160-5. PMID: 28855974; PMCID: PMC5568566.

34. Fijan S. Microorganisms with claimed probiotic properties: an overview of recent literature. Int J Environ Res Public Health 2014; 11(5): 4745-67. DOI: 10.3390/ijerph110504745. PMID: 24859749; PMCID: PMC4053917.

35. Nisha KK, Wasule DD. Probiotics in Skin Care Products- A Review. International Journal for Research Trends and Innovation 2018; 3(10): 166-175.

\section{Cite this article as:}

Disha P Prajapati and Tanvi R Dodiya. A Review on Skin microbiome: Novel Strategy in Cosmetics. Int. J. Res. Ayurveda Pharm. 2021;12(3):99-102 http://dx.doi.org/10.7897/2277$\underline{4343.120382}$ 